NASA Technical Memorandum 4139

\title{
Multilayer Theory for \\ Delamination Analysis \\ of a Composite Curved \\ Bar Subjected to End \\ Forces and End Moments
}

William L. Ko and Raymond H. Jackson

Ames Research Center

Dryden Flight Research Facility

Edwards, California

Office of Management

Scientific and Technical

Information Division 


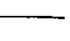




\section{SUMMARY}

A composite test specimen in the shape of a semicircular curved bar subjected to bending offers an excellent stress field for studying the open-mode delamination behavior of laminated composite materials. This is because the open-mode delamination nucleates at the midspan of the the curved bar. The classical anisotropic elasticity theory was used to construct a "multilayer" theory for the calculations of the stress and deformation fields induced in the multilayered composite semicircular curved bar subjected to end forces and end moments. The radial location and intensity of the open-mode delamination stress were calculated and were compared with the results obtained from the anisotropic continuum theory and from the finite element method. The multilayer theory gave more accurate predictions of the location and the intensity of the open-mode delamination stress than those calculated from the anisotropic continuum theory.

\section{NOMENCLATURE}

\begin{tabular}{|c|c|}
\hline$A, B, C, D$ & arbitrary constants associated with $F$ for loading case of end forces $P$ \\
\hline$A^{\prime}, B^{\prime}, C^{\prime}, D^{\prime}$ & arbitrary constants associated with $F$ for loading case of end moments $M$ \\
\hline $\bar{A}, \bar{B}, \bar{C}, \bar{D}$ & arbitrary constants associated with $F$ for loading case of end moments $M$ for isotropic materials \\
\hline$a$ & inner radius of semicircular curved bar \\
\hline$a_{i}$ & outer radius of $i$ th layer of semicircular curved bar \\
\hline$a_{m}$ & mean radius of semicircular curved bar, $\frac{1}{2}(a+b)$ \\
\hline$b$ & outer radius of semicircular curved bar \\
\hline$E_{L}$ & modulus of elasticity of single ply in fiber direction \\
\hline$E_{T}$ & modulus of elasticity of single-ply transverse to fiber direction \\
\hline$E_{r}$ & modulus of elasticity in $r$ direction \\
\hline$E_{\theta}$ & modulus of elasticity in $\theta$ direction \\
\hline$E 41$ & quadrilateral membrane element \\
\hline$e$ & loading axis offset \\
\hline$F$ & Airy stress function \\
\hline$G_{L T}$ & shear modulus of single ply \\
\hline$G_{r} \theta$ & shear modulus associated with $r-\theta$ system \\
\hline$h$ & width of semicircular curved bar \\
\hline$i$ & index associated with $i$ th layer, $i=1,2,3, \ldots N$ \\
\hline$k$ & anisotropic parameter, $\sqrt{\frac{E_{\theta}}{E_{r}}}$ \\
\hline$M$ & applied end moment \\
\hline$N$ & total number of laminated layers \\
\hline$P$ & applied end force \\
\hline$r$ & radial distance \\
\hline$r_{D}$ & radial location of $\sigma_{D}$ \\
\hline$r_{m}$ & radial location of $\left(\sigma_{\uparrow}\right)_{\max }$ \\
\hline$r_{m}^{\prime}$ & radial location of $\left(\sigma_{r}^{\prime}\right)_{\max }$ \\
\hline
\end{tabular}




$\begin{array}{ll}r_{0} & \text { radial location of zero } \sigma_{\theta} \\ u_{r} & \text { displacement in } r \text { direction } \\ u_{\theta} & \text { displacement in } \theta \text { direction } \\ x, y & \text { rectangular Cartesian coordinates } \\ \beta & \text { anisotropic parameter, } \sqrt{1+\frac{E_{\theta}}{E_{r}}\left(1-2 \nu_{r \theta}\right)+\frac{E_{\theta}}{G_{r \theta}}} \\ \gamma_{r \theta} & \text { shear strain in } r-\theta \text { plane } \\ \delta & \text { composite ply thickness } \\ \epsilon_{r} & \text { strain in } r \text { direction } \\ \epsilon_{\theta} & \text { strain in } \theta \text { direction } \\ \theta & \text { tangential coordinate } \\ \nu_{L T} & \text { Poisson ratio of single-ply composite } \\ \nu_{r \theta}, \nu_{\theta r} & \text { Poisson ratios } \\ \nu_{r z}, \nu_{z r} & \\ \nu_{\theta z}, \nu_{z \theta} & \\ \sigma_{D} & \text { delamination stress in C-coupon } \\ \sigma_{r} & \text { radial stress } \\ \left(\sigma_{r}\right)_{\max } & \text { delamination stress for the case of end forces } P, \sigma_{r}\left(r_{m}, \frac{\pi}{2}\right) \\ \left(\sigma_{r}^{\prime}\right)_{\max } & \text { delamination stress for the case of end moments } M, \sigma_{r}\left(r_{m}^{\prime}\right) \\ \sigma_{\theta} & \text { tangential stress } \\ \tau_{r \theta} & \text { shear stress } \\ {[]^{(i)}} & \text { quantity associated with } i \text { th layer } \\ {[]_{i}} & \text { quantity associated with } i \text { th layer }\end{array}$

\section{INTRODUCTION}

One of the major causes of stiffness and strength degradations in laminated composite structures is the delaminations between composite layers. In most engineering applications, laminated composite structures have certain curvatures (for example, curved panels and curved beams). If the curved composite structure is subjected to bending that tends to flatten the composite structure, tensile stresses can be generated in the thickness direction of the composites. Also, shear stresses could be induced if the bending is not a "pure" bending. Under normal operations, if the above type of bending occurs cyclically, open-mode delaminations or shear-mode delaminations could nucleate at the sites of pcak interlaminar tensile stresses or at the sites of peak interlaminar shear stresses. Continuation of these bending cyclings will cause the delamination zones to grow in size and ultimately cause the composite structures to lose their structural integrity (loss of stiffness and strength) due to excessive delaminations. The type of delamination failure (open mode or shear mode) depends on which type of interlaminar strength (tensile or shear) is reached first.

One of the most appealing geometries of a fatigue test coupon for studying the composite delamination phenomenon is the semicircular curved bar shape (C-coupon). When such a test specimen is subjected to end forces (that is, nonpure bending), the peak radial stress (tension, if the bending tends to increase the radius of the curvature of the curved bar) and the peak shear stress induced in the curved bar will be identical in magnitudes but are out of phase in the tangential direction by $\frac{\pi}{2} \cdot{ }^{1}$ Namely, the peak radial stress is located at the midspan point of the semicircular curved bar, but the peak shear stresses occur at both ends of the semicircular curved bar. The radial distance of both 
the peak radial and the peak shear stresses are exactly the same. ${ }^{1}$ The above nature of the semicircular curved bar offers an excellent situation for studying the initiation and subsequent propagation of delamination zones (open-mode or shear-mode) under cyclic loadings and for studying the fatigue behavior (degradation of stiffness and strength) of multilayered composite materials. In reference 1, Ko represented the multilayered composite semicircular bar with an equivalent continuous anisotropic material, and calculated radial locations and intensities of peak radial stresses induced in the curved bar subjected to end forces and end moments. Radial location and intensity of peak radial stress were calculated for different curved bar geometries (ratios of outer and inner radii) and for different degrees of anisotropy. Tolf ${ }^{2}$ also conducted stress analysis of curved laminated beams using both continuous and discrete theories. He considered only the "pure" bending case. In this paper the multilayer theory (discrete theory instead of continuous theory) and the finite element method were used to perform similar delamination analysis of the multilayered semicircular composite curved bar subjected to end forces and end moments. The resulting predictions of locations and intensities of peak radial stresses are compared with the results of the anisotropic continuum theory presented in reference 1 .

\section{COMPOSITE CURVED BAR}

Figure 1 shows the geometry of the composite curved bar (C-coupon) for delamination fatigue tests of composite materials. Because finite areas are needed for the load application points, both ends of the curved bar must be extended slightly. Thus, the C-coupon consists of a semicircular curved region with straight regions at both ends. Under the application of end forces $P$, the loading axis will have certain offset $e$ from the vertical diameter of the semicircle. Thus, the loading condition on the C-coupon is the summation of the following two loading conditions (sce fig. 2): (1) end forces $P$ at the ends of the semicircle and (2) end moment $M=P e$ at the ends of the semicircle.

Because the interface between $0^{\circ}$ and $90^{\circ}$ composite plies has the highest Poisson's ratio mismatch in laying up the composite plies for fabricating the C-coupon, it is desirable to place the $90^{\circ}$ or angle plies at the peak radial stress point to ensure that the delamination will nucleate at that point. Because of this demand, the precise location of the peak radial stress point must be known. The following sections will show how to determine the intensities and radial locations of peak radial stresses in the semicircular composite curved bar.

\section{ANISOTROPIC CONTINUUM THEORY}

For bending a linearly elastic continuous curved bar with cylindrical anisotropy, the Airy stress function $F$, written in cylindrical coordinate system, takes on the following functional forms: ${ }^{3}$

(a) for end forces $P$ (fig. 2b)

$$
F=\left[A r^{1+\beta}+B r^{1-\beta}+C r+D r \ln r\right] \sin \theta
$$

(b) for end moments $M$ (fig. 2c)

$$
F=A^{\prime}+B^{\prime} r^{2}+C^{\prime} r^{1+k}+D^{\prime} r^{1-k}
$$

where $\{A, B, C, D\}$ and $\left\{A^{\prime}, B^{\prime}, C^{\prime}, D^{\prime}\right\}$ are arbitrary constants that must be determined from boundary conditions, and the two anisotropic parameters $\beta$ and $k$ are respectively defined as

$$
\begin{aligned}
& \beta \equiv \sqrt{1+\frac{E_{\theta}}{E_{\tau}}\left(1-2 \nu_{r \theta}\right)+\frac{E_{\theta}}{G_{r \theta}}} \\
& k \equiv \sqrt{\frac{E_{\theta}}{E_{r}}}
\end{aligned}
$$

For the isotropic case, $\beta=2$ and $k=1$. 
The functional form given in equation (1) is also applicable to the isotropic case $(\beta=2)$. However, the functional format given in equation (2) cannot be applied directly to the isotropic case by simply setting $k=1$. For the isotropic case, equation (2) must be expanded in the neighborhood of $k=1$ using the relationship $\ln r^{ \pm(k-1)} \approx r^{ \pm(k-1)}-1$ where $k-1 \ll 1$ to the following familiar form ${ }^{4}$

$$
F=\bar{A}+\bar{B} r^{2}+\bar{C} r^{2} \ln r+\bar{D} \ln r
$$

where $\{\bar{A}, \bar{B}, \bar{C}, \bar{D}\}$ are a different set of arbitrary constants.

Stresses in the cylindrically anisotropic body may be expressed in terms of the stress function $F$ as

$$
\begin{aligned}
\sigma_{r} & =\frac{1}{r} \frac{\partial F}{\partial r}+\frac{1}{r^{2}} \frac{\partial^{2} F}{\partial \theta^{2}} \\
\sigma_{\theta} & =-\frac{\partial^{2} F}{\partial r^{2}} \\
\tau_{r \theta} & =-\frac{\partial^{2}}{\partial r \partial \theta}\left(\frac{F}{r}\right)
\end{aligned}
$$

and the stress-strain relationships for the plane stress case are given by

$$
\begin{aligned}
\epsilon_{r} & =\frac{1}{E_{r}} \sigma_{r}-\frac{\nu_{\theta r}}{E_{\theta}} \sigma_{\theta} \\
\epsilon_{\theta} & =-\frac{\nu_{r \theta}}{E_{r}} \sigma_{r}+\frac{1}{E_{\theta}} \sigma_{\theta} \\
\gamma_{r \theta} & =\frac{1}{G_{r \theta}} \tau_{r \theta}
\end{aligned}
$$

with the reciprocity relationship of

$$
\frac{\nu_{r \theta}}{E_{r}}=\frac{\nu_{\theta r}}{E_{\theta}}
$$

The strains are related to the displacements through the following formulae:

$$
\begin{aligned}
\epsilon_{\mathrm{r}} & =\frac{\partial u_{\mathrm{r}}}{\partial r} \\
\epsilon_{\theta} & =\frac{1}{r} \frac{\partial u_{\theta}}{\partial \theta}+\frac{u_{r}}{r} \\
\gamma_{r \theta} & =\frac{1}{2}\left(\frac{1}{r} \frac{\partial u_{r}}{\partial \theta}+\frac{\partial u_{\theta}}{\partial r}-\frac{u_{\theta}}{r}\right)
\end{aligned}
$$

\subsection{Stresses}

Substitution of equations (1) and (2) into equations (6) through (8) yields the following stress equations in terms of the unknown arbitrary constants $\{A, B, D\}$ or $\left\{B^{\prime}, C^{\prime}, D^{\prime}\right\}$ :

*For the plane strain case, $E_{r}, E_{\theta}, \nu_{\theta r}$, and $\nu_{r \theta}$ are simply replaced with $\frac{E_{r}}{1-\nu_{z r} \nu_{r z}}, \frac{E_{\theta}}{1-\nu_{z \theta} \nu_{\theta z}}, \frac{\nu_{\theta r}}{1-\nu_{z \theta} \nu_{\theta z}}\left(1+\frac{\nu_{r z} \nu_{\theta z}}{\nu_{\theta r}}\right)$ and $\frac{\nu_{z \theta}}{1-\nu_{z r} \nu_{r z}}\left(1+\frac{\nu_{z \theta} \nu_{r z}}{\nu_{r \theta}}\right)$, respectively. 
(a) for end forces $P$

$$
\begin{aligned}
& \sigma_{r}(r, \theta)=\left[A \beta r^{\beta-1}-B \beta r^{-\beta-1}+\frac{D}{r}\right] \sin \theta \\
& \sigma_{\theta}(r, \theta)=\left[A \beta(1+\beta) r^{\beta-1}-B \beta(1-\beta) r^{-\beta-1}+\frac{D}{r}\right] \sin \theta \\
& \tau_{r \theta}(r, \theta)=-\left[A \beta r^{\beta-1}-B \beta r^{-\beta-1}+\frac{D}{r}\right] \cos \theta
\end{aligned}
$$

(b) for end moments $M$

$$
\begin{aligned}
\sigma_{r}(r) & =2 B^{\prime}+C^{\prime}(1+k) r^{k-1}+D^{\prime}(1-k) r^{-k-1} \\
\sigma_{\theta}(r) & =2 B^{\prime}+C^{\prime} k(1+k) r^{k-1}-D^{\prime} k(1-k) r^{-k-1} \\
\tau_{r \theta} & =0
\end{aligned}
$$

Notice that the magnitudes of $\sigma_{\tau}$ (equation (16)) and $\tau_{r \theta}$ (equation (18)) for the case of end forces $P$ are identical, but are out of phase in the $\theta$ direction by $\frac{\pi}{2}$.

\subsection{Displacements}

Using equations (9) through (12), (16) through (18), and (19) through (21), the displacements $u_{r}$ (equation (13)) and $u_{\theta}$ (equation (14)) may be integrated to give the following forms, neglecting the rigid body motion terms:

(a) for end forces $P$

$$
\begin{aligned}
u_{r}(r, \theta)=\{ & A r^{\beta}\left[\frac{1}{E_{\tau}}-(1+\beta) \frac{\nu_{\theta r}}{E_{\theta}}\right]+B r^{-\beta}\left[\frac{1}{E_{\tau}}-(1-\beta) \frac{\nu_{\theta r}}{E_{\theta}}\right] \\
& \left.+D(\ln r)\left(\frac{1}{E_{\tau}}-\frac{\nu_{\theta r}}{E_{\theta}}\right)\right\} \sin \theta \\
u_{\theta}(r, \theta)= & \left\{A r^{\beta}\left[\frac{1}{E_{\tau}}-\beta(1+\beta) \frac{1}{E_{\theta}}-\frac{\nu_{\theta r}}{E_{\theta}}\right]+B r^{-\beta}\left[\frac{1}{E_{\tau}}+\beta(1-\beta) \frac{1}{E_{\theta}}-\frac{\nu_{\theta r}}{E_{\theta}}\right]\right. \\
& \left.+D\left[(\ln r)\left(\frac{1}{E_{r}}-\frac{\nu_{\theta \tau}}{E_{\theta}}\right)-\left(\frac{1}{E_{\theta}}-\frac{\nu_{\theta r}}{E_{\theta}}\right)\right]\right\} \cos \theta
\end{aligned}
$$

(b) for end moments $M$

$$
\begin{aligned}
u_{r}(r)= & B^{\prime}\left\{2 r\left(\frac{1}{E_{\tau}}-\frac{\nu_{\theta r}}{E_{\theta}}\right)\right\}+C^{\prime}\left\{(1+k) r^{k}\left(\frac{1}{k} \frac{1}{E_{r}}-\frac{\nu_{\theta r}}{E_{\theta}}\right)\right\} \\
& -D^{\prime}\left\{(1-k) r^{-k}\left(\frac{1}{k} \frac{1}{E_{\tau}}+\frac{\nu_{\theta r}}{E_{\theta}}\right)\right\} \\
u_{\theta}(r, \theta)= & B^{\prime}\left\{2 r\left(\frac{1}{E_{\theta}}-\frac{1}{E_{\tau}}\right)\right\} \theta
\end{aligned}
$$

\subsection{Delamination Stresses and Their Locations}

For the continuous (or single layer) curved bar, the two sets of unknown constants $\{A, B, D\}$ and $\left\{B^{\prime}, C^{\prime}, D^{\prime}\right\}$ can be determined explicitly from the boundary conditions to give closed-form expressions for the stresses (equations (16) to (21)) and the displacements (equations (22) to (25)). ${ }^{1,3}$ By using the extreme condition $\frac{\partial}{\partial r} \sigma_{r}=0$, the functional expressions for the delamination stress (maximum value of $\sigma_{r}$ ) and its radial location were derived in reference 1 for both of the aforementioned loading cases. 


\section{MULTILAYER THEORY}

Figure 3 shows the multilayer ( $N$-layers), semicircular curved bar subjected to both end forces $P$ and end moments $M$. The stress field and displacement field in each layer $i(i=1,2, \ldots N)$ for each loading case may be obtained from the results given in section 3 .

\subsection{Boundary Conditions}

At each interface between layers $i$ and $i+1(i=1,2, \ldots N-1)$, the following boundary conditions for the continuities of stresses and displacements must hold (no sliding between layers).

(a) for end forces $P$

at $r=a_{i}$ :

$$
\begin{aligned}
& \sigma_{r}^{(i)}\left(a_{i}, \theta\right)=\sigma_{r}^{(i+1)}\left(a_{i}, \theta\right) \\
& \tau_{r \theta}^{(i)}\left(a_{i}, \theta\right)=\tau_{r \theta}^{(i+1)}\left(a_{i}, \theta\right) \\
& u_{r}^{(i)}\left(a_{i}, \theta\right)=u_{r}^{(i+1)}\left(a_{i}, \theta\right) \\
& u_{\theta}^{(i)}\left(a_{i}, \theta\right)=u_{\theta}^{(i+1)}\left(a_{i}, \theta\right)
\end{aligned}
$$

The boundary conditions at the traction-free inner surface $(i-1=0)$ and outer surface $(i=N)$ of the curved bar are

at $r=a_{0}=a$ :

$$
\begin{aligned}
& \sigma_{r}^{(1)}(a, \theta)=0 \\
& \tau_{r \theta}^{(1)}(a, \theta)=0
\end{aligned}
$$

at $r=a_{N}=b$ :

$$
\begin{aligned}
& \sigma_{r}^{(N)}(b, \theta)=0 \\
& \tau_{r \theta}^{(N)}(b, \theta)=0
\end{aligned}
$$

(b) for end forces $M$

at $r=a_{i}$ :

$$
\begin{aligned}
& \sigma_{r}^{(i)}\left(a_{i}\right)=\sigma_{r}^{(i+1)}\left(a_{i}\right) \\
& u_{r}^{(i)}\left(a_{i}\right)=u_{r}^{(i+1)}\left(a_{i}\right) \\
& u_{\theta}^{(i)}\left(a_{i}\right)=u_{\theta}^{(i+1)}\left(a_{i}\right)
\end{aligned}
$$

at $r=a_{0}=a$ :

$$
\sigma_{r}^{(1)}(a)=0
$$

at $r=a_{n}=b$ :

$$
\sigma_{r}^{(N)}(b)=0
$$


As mentioned earlier, $\sigma_{\tau}$ and $\tau_{r \theta}$ (equations (16) and (18)) for the case of end forces $P$ have identical $r$ dependency. Thus, if $\sigma_{\tau}$ satisfies the boundary conditions, $\tau_{r} \theta$ will also satisfy the boundary conditions automatically. Therefore, the boundary conditions associated with $\tau_{r \theta}$ (equations (27), (31), and (33)) are not needed.

For each loading case, each set of the previous boundary conditions will give $2+3(N-1)=3 N-1$ equations for determining the $3 N$ unknowns $A_{i}, B_{i}, D_{i}(i=1,2, \ldots N)$ for the case of end forces $P$, or $3 N$ unknowns $B_{i}^{\prime}, C_{i}^{\prime}, D_{i}^{\prime}(i=1,2, \ldots N)$ for the case of end moments $M$.

The last equation needed for each loading case is the condition that the end force $P$ or the end moment $M$ is balanced by the stresses in the curved bar:

(a) for end forces $P$

$$
-P=\sum_{i=1}^{N} \int_{a_{i-1}}^{a_{i}} T_{r \theta}^{(i)}(r, 0) d r \quad ; \quad \theta=0
$$

(b) for end moments $M$

$$
-M=\sum_{i=1}^{N} \int_{a_{i-1}}^{a_{i}}\left(r-r_{0}\right) \sigma_{\theta}(r) d r
$$

where the negative signs in front of $P$ and $M$ are to increase the radius of curvature of the curved bar, and $r_{0}$ is the unknown radial location where $\sigma_{\theta}=0$. For pure bending we have

$$
\sum_{i=1}^{N} \int_{a_{i-1}}^{a_{i}} \sigma_{\theta}(r) d r=0
$$

Therefore (since $r_{0}$ term vanishes), equation (40) becomes:

$$
-M=\sum_{i=1}^{N} \int_{a_{i-1}}^{a_{i}} r \sigma_{\theta}(r) d r
$$

\subsection{Boundary Conditions in Final Forms}

After substitution of stress and displacement expressions given respectively in sections 3.1 and 3.2 into the boundary conditions given in section 4.1, the following final forms of the boundary conditions are obtained:

(a) for end forces $P$

for $\sigma_{r}^{(1)}$ (equation (30)):

$$
A_{1} \beta_{1} a^{\beta_{1}}-B_{1} \beta_{1} a^{-\beta_{1}}+D_{1}=0
$$

for $\sigma_{r}^{(i)}$ (equation (26)):

$$
A_{i} \beta_{i} a_{i}^{\beta_{i}}-B_{i} \beta_{i} a_{i}^{-\beta_{i}}+D_{i}-A_{i+1} \beta_{i+1} a_{i}^{\beta_{i+1}}+B_{i+1} \beta_{i+1} a_{i}^{-\beta_{i+1}}-D_{i+1}=0
$$

for $\sigma_{\tau}^{(N)}$ (equation (32)):

$$
A_{N} \beta_{N} a_{N}^{\beta_{N}}-B_{N} \beta_{N} a_{N}^{-\beta_{N}}+D_{N}=0
$$


for $u_{r}^{(i)}$ (equation (28)):

$$
\begin{aligned}
& A_{i} a_{i}^{\beta_{i}}\left\{\frac{1}{E_{r}^{(i)}}-\left(1+\beta_{i}\right) \frac{\nu_{\theta r}^{(i)}}{E_{\theta}^{(i)}}\right\}+B_{i} a_{i}^{-\beta_{i}}\left\{\frac{1}{E_{r}^{(i)}}-\left(1-\beta_{i}\right) \frac{\nu_{\theta r}^{(i)}}{E_{\theta}^{(i)}}\right\} \\
&+ D_{i}\left(\ln a_{i}\right)\left(\frac{1}{E_{r}^{(i)}}-\frac{\nu_{\theta r}^{(i)}}{E_{\theta}^{(i)}}\right) \\
&-A_{i+1} a_{i}^{\beta_{i+1}}\left\{\frac{1}{E_{r}^{(i+1)}}-\left(1+\beta_{i+1}\right) \frac{\nu_{\theta r}^{(i+1)}}{E_{\theta}^{(i+1)}}\right\}-B_{i+1} a_{i}^{-\beta_{i+1}}\left\{\frac{1}{E_{r}^{(i+1)}}-\left(1-\beta_{i+1}\right) \frac{\nu_{\theta r}^{(i+1)}}{E_{\theta}^{(i+1)}}\right\} \\
&-D_{i+1}\left(\ln a_{i}\right)\left(\frac{1}{E_{r}^{(i+1)}}-\frac{\nu_{\theta r}^{(i+1)}}{E_{\theta}^{(i+1)}}\right)=0
\end{aligned}
$$

for $u_{\theta}^{(i)}$ (equation (29)):

$$
\begin{aligned}
A_{i} a_{i}^{\beta_{i}} \frac{\beta_{i}}{E_{\theta}^{(i)}}\left\{\left(1+\beta_{i}\right)-\nu_{\theta r}^{(i)}\right\}- & B_{i} a_{i}^{-\beta_{i}} \frac{\beta_{i}}{E_{\theta}^{(i)}}\left\{\left(1-\beta_{i}\right)-\nu_{\theta r}^{(i)}\right\} \\
& +D_{i} \frac{1}{E_{\theta}^{(i)}}\left(1-\nu_{\theta r}^{(i)}\right) \\
-A_{i+1} a_{i}^{\beta_{i+1}} \frac{\beta_{i+1}}{E_{\theta}^{(i+1)}}\left\{\left(1+\beta_{i+1}\right)-\right. & \left.\nu_{\theta r}^{(i+1)}\right\}+B_{i+1} a_{i}^{-\beta_{i+1}} \frac{\beta_{i+1}}{E_{\theta}^{(i+1)}}\left\{\left(1-\beta_{i+1}\right)-\nu_{\theta r}^{(i+1)}\right\} \\
& -D_{i+1} \frac{1}{E_{\theta}^{(i+1)}}\left(1-\nu_{\theta r}^{(i+1)}\right)=0
\end{aligned}
$$

Equation (47) was obtained by taking the difference between the boundary conditions (28) and (29). This was done because the resulting expression (47) is simpler than using equation (29).

for $P$ (equation (39)):

$$
\sum_{i=1}^{N}\left[A_{i}\left(a_{i}^{\beta_{i}}-a_{i-1}^{\beta_{i}}\right)+B_{i}\left(a_{i}^{-\beta_{i}}-a_{i-1}^{-\beta_{i}}\right)+D_{i}\left(\ln a_{i}-\ln a_{i-1}\right)\right]=P
$$

(b) for end moments $M$

for $\sigma_{r}^{(1)}$ (equation (37)):

$$
2 B_{1}^{\prime}+C_{1}^{\prime}\left(1+k_{1}\right) a^{k_{1}-1}+D_{1}^{\prime}\left(1-k_{1}\right) a^{-k_{1}-1}=0
$$

for $\sigma_{r}^{(i)}$ (equation (34)):

$$
\begin{gathered}
2 B_{i}^{\prime}+C_{i}^{\prime}\left(1+k_{i}\right) a_{i}^{k_{i}-1}+D_{i}^{\prime}\left(1-k_{i}\right) a_{i}^{-k_{i}-1} \\
-2 B_{i+1}^{\prime}-C_{i+1}^{\prime}\left(1+k_{i+1}\right) a_{i}^{k_{i+1}-1}-D_{i+1}^{\prime}\left(1-k_{i+1}\right) a_{i}^{-k_{i+1}-1}=0
\end{gathered}
$$

for $\sigma_{r}^{(N)}$ (equation (38)):

$$
2 B_{N}^{\prime}+C_{N}^{\prime}\left(1+k_{N}\right) b^{k_{N}-1}+D_{N}^{\prime}\left(1-k_{N}\right) b^{-k_{N}-1}=0
$$


for $u_{r}^{(i)}$ (equation (35)):

$$
\begin{aligned}
& B_{i}^{\prime}\left\{2 a_{i}\left(\frac{1}{E_{r}^{(i)}}-\frac{\nu_{\theta r}^{(i)}}{E_{\theta}^{(i)}}\right)\right\}+C_{i}^{\prime}\left\{\left(1+k_{i}\right) a_{i}^{k_{i}}\left(\frac{1}{k_{i}} \frac{1}{E_{r}^{(i)}}-\frac{\nu_{\theta r}^{(i)}}{E_{\theta}^{(i)}}\right)\right\} \\
&-D_{i}^{\prime}\left\{\left(1-k_{i}\right) a_{i}^{-k_{i}}\left(\frac{1}{k_{i}} \frac{1}{E_{r}^{(i)}}+\frac{\nu_{\theta r}^{(i)}}{E_{\theta}^{(i)}}\right)\right\} \\
&-B_{i+1}^{\prime}\left\{2 a_{i}\left(\frac{1}{E_{r}^{(i+1)}}-\frac{\nu_{\theta r}^{(i+1)}}{E_{\theta}^{(i+1)}}\right)\right\}-C_{i+1}^{\prime}\left\{\left(1+k_{i+1}\right) a_{i}^{k_{i+1}}\left(\frac{1}{k_{i+1}} \frac{1}{E_{r}^{(i+1)}}-\frac{\nu_{\theta r}^{(i+1)}}{E_{\theta}^{(i+1)}}\right)\right\} \\
&+D_{i+1}^{\prime}\left\{\left(1-k_{i+1}\right) a_{i}^{-k_{i+1}}\left(\frac{1}{k_{i+1}} \frac{1}{E_{r}^{(i+1)}}+\frac{\nu_{\theta r}^{(i+1)}}{E_{\theta}^{(i+1)}}\right)\right\}=0
\end{aligned}
$$

for $u_{\theta}^{(i)}$ (equation (36)):

$$
B_{i}^{\prime}\left\{2 a_{i}\left(\frac{1}{E_{\theta}^{(i)}}-\frac{1}{E_{\tau}^{(i)}}\right)\right\}-B_{i+1}^{\prime}\left\{2 a_{i}\left(\frac{1}{E_{\theta}^{(i+1)}}-\frac{1}{E_{r}^{(i+1)}}\right)\right\}=0
$$

for $M$ (equation (42)):

$$
\sum_{i=1}^{N}\left[B_{i}^{\prime}\left(a_{i}^{2}-a_{i-1}^{2}\right)+C_{i}^{\prime} k_{i}\left(a_{i}^{k_{i}+1}-a_{i-1}^{k_{i}+1}\right)-D_{i}^{\prime} k_{i}\left(a_{i}^{-k_{i}+1}-a_{i-1}^{-k_{i}+1}\right)\right]=-M
$$

\subsection{Delamination Stresses and Their Locations}

At exactly which layer the value of $\sigma_{r}$ for each loading case will become maximum cannot be predicted until after all the unknown arbitrary constants $\left\{A_{i}, B_{i}, C_{i}\right\}$ or $\left\{B_{i}^{\prime}, C_{i}^{\prime}, D_{i}^{\prime}\right\}$ are determined from the appropriate boundary conditions given in section 4.2. Suppose $\left(\sigma_{r}\right)_{\max }$ (or $\left(\sigma_{r}^{\prime}\right)_{\max }$ ), the maximum value of $\sigma_{r}$ due to end forces $P$ (or end moments $M$ ), occurs in the $i$ th layer, then by using the extreme condition $\frac{\partial}{\partial r} \sigma_{r}=0$, the radial location $r_{m}$ (or $r_{m}^{\prime}$ ) of $\left(\sigma_{r}\right)_{\max }$ (or $\left(\sigma_{r}^{\prime}\right)_{\max }$ ) may be calculated from equation (16) (or equation (19)) as:

(a) for end forces $P$

$$
r_{m}=\left[\frac{D_{i}-\sqrt{D_{i}^{2}-4 A_{i} B_{i} \beta_{i}^{2}\left(\beta_{i}^{2}-1\right)}}{2 A_{i} \beta_{i}\left(\beta_{i}-1\right)}\right]^{\frac{1}{\beta_{i}}}
$$

(b) for end moments $M$

$$
r_{m}^{\prime}=\left[-\frac{D_{i}^{\prime}}{C_{i}^{\prime}}\right]^{\frac{1}{2 k_{i}}}
$$

And the delamination stresses $\left(\sigma_{r}\right)_{\max }$ and $\left(\sigma_{r}^{\prime}\right)_{\max }$ for the two loading cases may be written as

(a) for end forces $P$

$$
\left(\sigma_{\tau}\right)_{\max } \equiv \sigma_{r}\left(r_{m}, \frac{\pi}{2}\right)=\left[A_{i} \beta_{i} r_{m}^{\beta_{i}-1}-B_{i} \beta_{i} r_{m}^{-\beta_{i}-1}+\frac{D_{i}}{r_{m}}\right]
$$


(b) for end moments $M$

$$
\left(\sigma_{r}^{\prime}\right)_{\max } \equiv \sigma_{r}\left(r_{m}^{\prime}\right)=2 B_{i}^{\prime}+C_{i}^{\prime}\left(1+k_{i}\right)\left(r_{m}^{\prime}\right)^{k_{i}-1}+D_{i}^{\prime}\left(1-k_{i}\right)\left(r_{m}^{\prime}\right)^{-k_{i}-1}
$$

\subsection{Delamination Stress in C-coupon}

The delamination stresses $\left(\sigma_{r}\right)_{\max }$ (due to $P$ ) and $\left(\sigma_{r}^{\prime}\right)_{\max }$ (due to $M$ ) do not occur at the same radial locations of the curved bar (see equations (55) and (56)). Thus, the delamination stress $\sigma_{D}$ in the C-coupon cannot be constructed by simply summing up $\left(\sigma_{\tau}\right)_{\max }$ and $\left(\sigma_{\tau}^{\prime}\right)_{\max }$. The value of $\sigma_{D}$ must be evaluated at $r=r_{D}$ where the summation of the radial stress $\sigma_{r}\left(r, \frac{\pi}{2}\right)$ due to $P$ and the radial stress $\sigma_{r}(r)$ due to $M$ become maximum. Namely,

$$
\sigma_{D}=\sigma_{r}\left(r_{D}, \frac{\pi}{2}\right)+\sigma_{r}\left(r_{D}\right)
$$

where, the value of $r_{D}$ is calculated from the following extreme condition:

$$
\frac{d}{d r}\left[\begin{array}{c}
\text { Due to } \mathrm{P} \\
\left.\sigma_{r}\left(r, \frac{\pi}{2}\right)+\sigma_{r}(r)\right]=0
\end{array}\right.
$$

which, after substitution of equations (16) and (19) and after performing differentiation, becomes

$$
\begin{aligned}
A_{i} \beta_{i}\left(\beta_{i}-1\right) r_{D}^{\beta_{i}}+B_{i} \beta_{i}\left(\beta_{i}+1\right) r_{D}^{-\beta_{i}}-D_{i} \\
+\left(k_{i}^{2}-1\right)\left(C_{i}^{\prime} r_{D}^{k_{i}}+D_{i}^{\prime} r_{D}^{-k_{i}}\right)=0
\end{aligned}
$$

As will be seen later, the radial location $r_{D}$ of the delamination stress $\sigma_{D}$ in the C-coupon is somewhere between $r_{m}$ and $r_{m}^{\prime}$ (that is $r_{m}<r_{D}<r_{m}^{\prime}$ ).

\section{NUMERICAL EXAMPLES}

The anisotropic continuum theory and the multilayer theory presented respectively in sections 3 (or ref. 1) and 4 will now be applied to the delamination analysis of the composite C-coupon. One type of composite C-coupon under development has the following geometry and ply properties:

$\begin{array}{ll}\text { inner radius } & \mathrm{a}=2.1590 \mathrm{~cm}(0.85 \mathrm{in} .) \\ \text { outer radius } & \mathrm{b}=2.9724 \mathrm{~cm}(1.17022 \mathrm{in} .) \\ \text { loading axis offset } & \mathrm{e}=0.9525 \mathrm{~cm}(0.375 \mathrm{in} .) \\ \text { width } & \mathrm{h}=2.54 \mathrm{~cm}(1 \mathrm{in} .) \\ \text { ply thickness } & \delta=0.01506 \mathrm{~cm}(0.00593 \mathrm{in} .) \\ \text { mean radius } & a_{m}=\frac{a+b}{2}=2.5657 \mathrm{~cm}(1.01011 \mathrm{in} .) \\ \text { radii ratio } & \frac{b}{a}=1.3767 \\ & E_{L}=17.2369 \times 10^{10} \mathrm{~N} / \mathrm{m}^{2}\left(25 \times 10^{6} \mathrm{lb} / \mathrm{in}^{2}\right) \\ & E_{T}=0.8274 \times 10^{10} \mathrm{~N} / \mathrm{m}^{2}\left(1.2 \times 10^{6} \mathrm{lb} / \mathrm{in}^{2}\right) \\ & G_{L T}=0.4137 \times 10^{10} \mathrm{~N} / \mathrm{m}^{2}\left(0.6 \times 10^{6} \mathrm{lb} / \mathrm{in} .{ }^{2}\right) \\ & \nu_{L T}=0.33 \\ & \nu_{T L}=0.01584\end{array}$


The aforementioned $\mathrm{C}$-coupon has 54 composite plies having the stacking sequence of $\left[00^{\circ} /+15^{\circ} /-15^{\circ} /-\right.$ $\left.15 \%+15^{\circ} / 0_{25}^{\circ}\right]$.

\subsection{Equivalent Continuum}

In order to apply the anisotropic continuum theory, the laminated composite C-coupon will be represented by an equivalent anisotropic continuum having the following effective material properties:

$$
\begin{aligned}
E_{\theta} & =16.3220 \times 10^{10} \mathrm{~N} / \mathrm{m}^{2}\left(23.6731 \times 10^{6} \mathrm{lb} / \mathrm{in} .{ }^{2}\right) \\
E_{r} & =0.8274 \times 10^{10} \mathrm{~N} / \mathrm{m}^{2}\left(1.2 \times 10^{6} \mathrm{lb} / \mathrm{in}^{2}\right) \\
G_{r \theta} & =0.4137 \times 10^{10} \mathrm{~N} / \mathrm{m}^{2}\left(0.6 \times 10^{6} \mathrm{lb} / \mathrm{in} .{ }^{2}\right) \\
\nu_{r \theta} & \approx 0.01673
\end{aligned}
$$

Based on these effective material properties, the equivalent continuum representing the $\mathrm{C}$-coupon has the values of anisotropic parameters as $\beta=7.7151$ and $k=4.4416$.

\subsection{Multilayer System}

For the purpose of applying the multilayer theory to the C-coupon, the extended linear regions at both ends will be neglected, and only the semicircular region subjected to two types of loadings (end forces $P$ and end moments $M$, figure 2) will be considered. For simplification, each group of 25 layers of the $0^{\circ}$ plies will be represented by one layer of anisotropic continuum, and the center region of 4 layers of $\pm 15^{\circ}$ angle plies will be represented by another anisotropic continuum. Thus, the 54-layer composite will be represented by 3 layers of anisotropic continua.

(a) $0^{\circ}$ plies

The inner $(i=1)$ and the outer $(i=3)$ layers have the following effective material properties:

$$
\begin{aligned}
& E_{\theta}^{(i)}=E_{L}=17.2369 \times 10^{10} \mathrm{~N} / \mathrm{m}^{2}\left(25 \times 10^{6} \mathrm{lb} / \mathrm{in}^{2}\right) \\
& E_{r}^{(i)}=E_{T}=0.8274 \times 10^{10} \mathrm{~N} / \mathrm{m}^{2}\left(1.2 \times 10^{6} \mathrm{lb} / \mathrm{in} .{ }^{2}\right) \\
& G_{r \theta}^{(i)}=G_{L T}=0.4137 \times 10^{10} \mathrm{~N} / \mathrm{m}^{2}\left(0.6 \times 10^{6} \mathrm{lb} / \mathrm{in} .{ }^{2}\right) \\
& \nu_{r \theta}^{(i)}=0.01584
\end{aligned}
$$

which give the values of anisotropic parameters as $\beta_{1}=\beta_{3}=7.9272$ and $k_{1}=k_{3}=4.5644$.

(b) $\pm 15^{\circ}$ plies

The center layer $(i=2)$ has the following effective material properties:

$$
\begin{aligned}
& E_{\theta}^{(2)}=4.8873 \times 10^{10} \mathrm{~N} / \mathrm{m}^{2}\left(7.0884 \times 10^{6} \mathrm{lb} / \mathrm{in} .{ }^{2}\right) \\
& E_{r}^{(2)}=0.8274 \times 10^{10} \mathrm{~N} / \mathrm{m}^{2}\left(1.2 \times 10^{6} \mathrm{lb} / \mathrm{in}^{2}\right) \\
& G_{r \theta}^{(2)}=0.4137 \times 10^{10} \mathrm{~N} / \mathrm{m}^{2}\left(0.6 \times 10^{6} \mathrm{lb} / \mathrm{in}^{2}\right) \\
& \nu_{r \theta}^{(2)} \approx 0.05590
\end{aligned}
$$

which give the values of the anisotropic parameters as $\beta_{2}=4.2498$ and $k_{2}=2.4304$.

The $3 N(N=3)$ boundary conditions for the aforementioned 3-layer laminated system may be written in matrix forms (see appendix) for solving the $3 N$ unknown constants $A_{i}, B_{i}, D_{i}(i=1,2,3)$, for the case of end forces $P$, or for solving the other set of $3 N$ unknown constants $B_{i}^{\prime}, C_{i}^{\prime}, D_{i}^{\prime}(i=1,2,3)$, for the case of end moments $M$. 


\section{FINITE ELEMENT ANALYSIS}

To verify the solution accuracies of the analysis for which the actual loading condition of the C-coupon was represented with the superposition of two loading cases of the semicircular curved bar (see fig. 2), finite element stress analysis was performed on the semicircular curved bar (under two loading cases) and on the C-coupon using the structural performance and resizing (SPAR) finite element computer program. ${ }^{5}$ Figures 4 and 5 respectively show the SPAR finite element models set up for the semicircular curved bar and the C-coupon. Because of symmetry with respect to the $x$ axis, only the half span of the semicircular curved bar and the C-coupon were modeled. Both systems were first reduced to three-layer systems as defined in section 5.2. Then layers 1,2, and 3 were respectively modeled in 10,2 , and 10 layers of quadrilateral membrane $E 41$ elements in the $r$ direction. In the tangential direction, the quarter-circular region $\left(0 \leq \theta \leq \frac{\pi}{2}\right)$ of the two systems was modeled with $90 E 41$ elements. The extended region $(-e \leq x \leq 0)$ of the C-coupon was modeled with 25 layers of $E 41$ elements in the $x$ direction.

The $\theta=90^{\circ}$ plane for each model was allowed to move freely in the $x$ direction (shown with rollers in figs. 4 and 5) but not in the $y$ direction. At the upper end of each model, only one point lying in the middle surface $\left(\sigma_{\theta}=0\right.$ point was found to be very close to the middle surface of the curved bar) was constrained to move freely in the $y$ direction only (no movement in the $x$ direction). Thus, the end of each model could rotate freely (shown with only one roller, figs. 4 and 5). The applied force $P$ and the applied moment $M$ were represented respectively with the distributions of $\tau_{r \theta}(r, 0)^{\prime}$ and $\sigma_{\theta}(r)$ obtained from the multilayer analysis. The sizes of the two SPAR models are listed below:

\begin{tabular}{ccc}
\hline \hline & $\begin{array}{c}\text { Semicircular } \\
\text { curved bar }\end{array}$ & C-coupon \\
\hline JLOCs & 2093 & 2668 \\
$E 41$ elemients & 1980 & 2530 \\
\hline \hline
\end{tabular}

\section{RESULTS}

\subsection{Semicircular Curved Bar}

Figure 6 shows the distributions of $\sigma_{\uparrow}$ in the $\theta=\frac{\pi}{2}$ plane for the case of end forces $P$ calculated from different theories. The values of $\left(\sigma_{r}\right)_{\max }$ and $r_{m}$ calculated from different theories are indicated in the figure. The values of $\left(\sigma_{r}\right)_{\max }$ calculated from different theories are quite close, except its location $r_{m}$. The multilayer theory and the finite element method predicted close values of $r_{m}$. The $\left(\sigma_{r}\right)_{\max }$ site predicted from the anisotropic continuum theory is located slightly closer to the middle surface than the $\left(\sigma_{r}\right)_{\max }$ sites predicted from the multilayer theory and SPAR. The $\left(\sigma_{r}\right)_{\max }$ site for the isotropic material is closest to the middle surface and is always located between the middle surface and the $\left(\sigma_{r}\right)_{\max }$ site predicted from the anisotropic continuum theory. This can be seen more clearly from the plots shown in figure 7 .

The distance between the sites of $\left(\sigma_{r}\right)_{\max }$ predicted from the multilayer theory and the anisotropic continuum theory is

$$
\underset{\substack{\text { Anisotropic } \\ \text { continuum }}}{\left(r_{m}\right.}-\left(r_{m}\right)_{\text {Multilayer }}=(0.4158-0.3907)(b-a)=0.0203 \mathrm{~cm}(0.0080 \mathrm{in} .)
$$

which is 1.3554 times the single ply thickness of $0.01506 \mathrm{~cm}(0.00593 \mathrm{in}$.).

Figure 8 shows the distributions of $\sigma_{r}$ for the case of end moments $M$ calculated from different theories. Unlike the previous case, the values of $r_{m}^{\prime}$ and $\left(\sigma_{\tau}^{\prime}\right)_{\max }$ calculated from different theories are quite close, showing that the 
value of $r_{m}^{\prime}$ is quite insensitive to the theory used. The multilayer theory predicted the shortest distance of $r_{m}^{\prime}$ (that is, the $\left(\sigma_{\tau}^{\prime}\right)_{\max }$ site is closest to the inner boundary of the curved bar). The site of $\left(\sigma_{\tau}^{\prime}\right)_{\max }$ predicted from the anisotropic continuum theory always lies between the middle surface and the $\left(\sigma_{\tau}^{\prime}\right)_{\max }$ site, based on isotropic theory. This is shown in figure 9.

The distance between the sites of $\left(\sigma_{\tau}^{\prime}\right)_{\max }$ predicted from the multilayer theory and the anisotropic continuum theory is given below

$$
\underset{\substack{\text { Anisotropic } \\ \text { continuum }}}{\left(r_{m}^{\prime}\right.}-\left(r_{m}^{\prime}\right)_{\text {Multilayer }}=(0.4346-0.4327)(b-a)=0.001545 \mathrm{~cm}(0.000608 \mathrm{in} .)
$$

which is only 0.1025 times the single-ply thickness of $0.01506 \mathrm{~cm}(0.00593 \mathrm{in}$.), and is therefore, insignificant.

Figures 10 and 11 respectively show the deformed shapes of the semicircular curved bar subjected to end forces $P$ and end moments $M$. The dimensionless radial displacements $\frac{\underline{u}_{x}}{a}$ at $\theta=0$ and $\theta=\frac{\pi}{2}$ are shown in the figures.

Table 1 summarizes all the values of $\left(\sigma_{r}\right)_{\max },\left(\sigma_{r}^{\prime}\right)_{\max }, r_{m}$, and $r_{m}^{\prime}$ calculated from different theories.

Table 1. Intensities and locations of delamination stresses in semicircular curved bar.

\begin{tabular}{lcccc}
\hline \hline \multirow{2}{*}{ Theory } & \multicolumn{3}{c}{ End forces $P$} & \multicolumn{3}{c}{ End moments $M$} \\
\cline { 2 - 5 } & \multicolumn{4}{c}{ Item } \\
\cline { 2 - 5 } & $\frac{\frac{h(b-a)}{P}\left(\sigma_{r}\right)_{\max }}{\frac{\tau_{m}-a}{b-a}}$ & $\frac{h a_{m}(b-a)}{M}\left(\sigma_{\mathrm{r}}^{\prime}\right)_{\max }$ & $\frac{r_{m}^{\prime}-a}{b-a}$ \\
\hline Anisotropic continuum & 1.4817 & 0.4158 & 1.5095 & 0.4346 \\
Multilayer theory & 1.4864 & 0.3907 & 1.4988 & 0.4327 \\
Isotropic continuum & 1.5135 & 0.4205 & 1.5222 & 0.4338 \\
SPAR & 1.4816 & 0.3935 & 1.4979 & 0.4398 \\
\hline \hline
\end{tabular}

\subsection{C-Coupon}

In order to determine the radial location $r_{D}$ and magnitude of delamination stress $\sigma_{D}$ for the C-coupon, the two radial stresses calculated from the semicircular curved bar due to $P$ and $M$ were summed up. The results are shown in figure 12 for multilayer, anisotropic, and isotropic cases. Notice that the radial location $r_{D}$ predicted from all three theories lie between $r_{m}$ and $r_{m}^{\prime}$ but closer to $r_{m}$ instead of $r_{m}^{\prime}$ because the stress contribution due to $P$ is larger than that due to $M\left(e<a_{m}\right)$. The distance between the locations of $\sigma_{D}$ predicted from multilayer and anisotropic continuum theories is about 1.05 times the ply thickness. The finite element solution data points obtained from the C-coupon model lie in the vicinity of the two curves obtained from the multilayer and anisotropic continuum theories. The intensity and the radial location of $\sigma_{D}$ predicted from SPAR (C-coupon) are closest to those predicted from the multilayer theory. Table 2 summarizes the values of $\sigma_{D}$ and $r_{D}$ predicted from different theories.

Table 2. Intensities and location of delamination stresses in C-coupon

\begin{tabular}{lcc}
\hline \hline \multirow{2}{*}{ Theory } & \multicolumn{2}{c}{ C-coupon under end forces $P$} \\
\cline { 2 - 3 } & $\frac{h(b-a)}{P} \sigma_{D}$ & $\frac{\tau D-a}{b-a}$ \\
\hline Anisotropic continuum & 2.0415 & 0.4212 \\
Multilayer theory & 2.0399 & 0.4017 \\
Isotropic continuum & 2.0783 & 0.4241 \\
SPAR (semicircular curved bar) & 2.0353 & 0.3935 \\
SPAR (C-coupon) & 2.0405 & 0.3935 \\
\hline \hline
\end{tabular}


Figure 13 shows the deformed shape of the C-coupon subjected to end forces $P$. The dimensionless radial displacement $\frac{u_{x}}{a}$ at midspan and at the free end are shown in the figure.

\title{
8 CONCLUSIONS
}

The multilayer theory was developed for delamination analysis of a semicircular composite curved bar subjected to end forces and end moments. The difference between the radial locations of the delamination stress (maximum radial stress) predicted from the multilayer theory and from the anisotropic continuum theory was approximately 1.4 times the ply thickness for the case of end forces and about $1 / 10$ of the ply thickness for the case of end moments. The superposition method (namely, by summing up the two radial stresses induced in the semicircular curved bar subjected to end forces and end moments), used to construct the delamination stress in the C-coupon, gave reasonably accurate intensity of the delamination stress for the $\mathrm{C}$-coupon. The finite element analysis of the $\mathrm{C}$-coupon gave the

radial location of the delamination stress in the $\mathrm{C}$-coupon much closer to that predicted from the multilayer theory than from the anisotropic continuum theory.

\author{
Ames Research Center \\ Dryden Flight Research Facility \\ National Aeronautics and Space Administration \\ Edwards, California, December 14, 1988
}

\section{REFERENCES}

1. Ko, W.L., Delamination Stresses in Semicircular Laminated Composite Bars, NASA TM-4026, 1988.

2. Tolf, G., "Stresses in a Curved Laminated Beam," Fiber Science and Technology, Vol. 19, No. 4, 1983, pp. 243-267.

3. Lekhnitskii, S.G., Anisotropic Plates, Gordon and Breach Science Publishers, New York, 1968.

4. Fung, Y.C., Foundations of Solid Mechanics, Prentice-Hall, Inc., Englewood Cliffs, New Jersey, 1965.

5. Whetstone, W.D., SPAR Structural Analysis System Reference Manual, System Level 13A, Vol. 1, Program Execution, NASA CR-158970-1, 1978. 


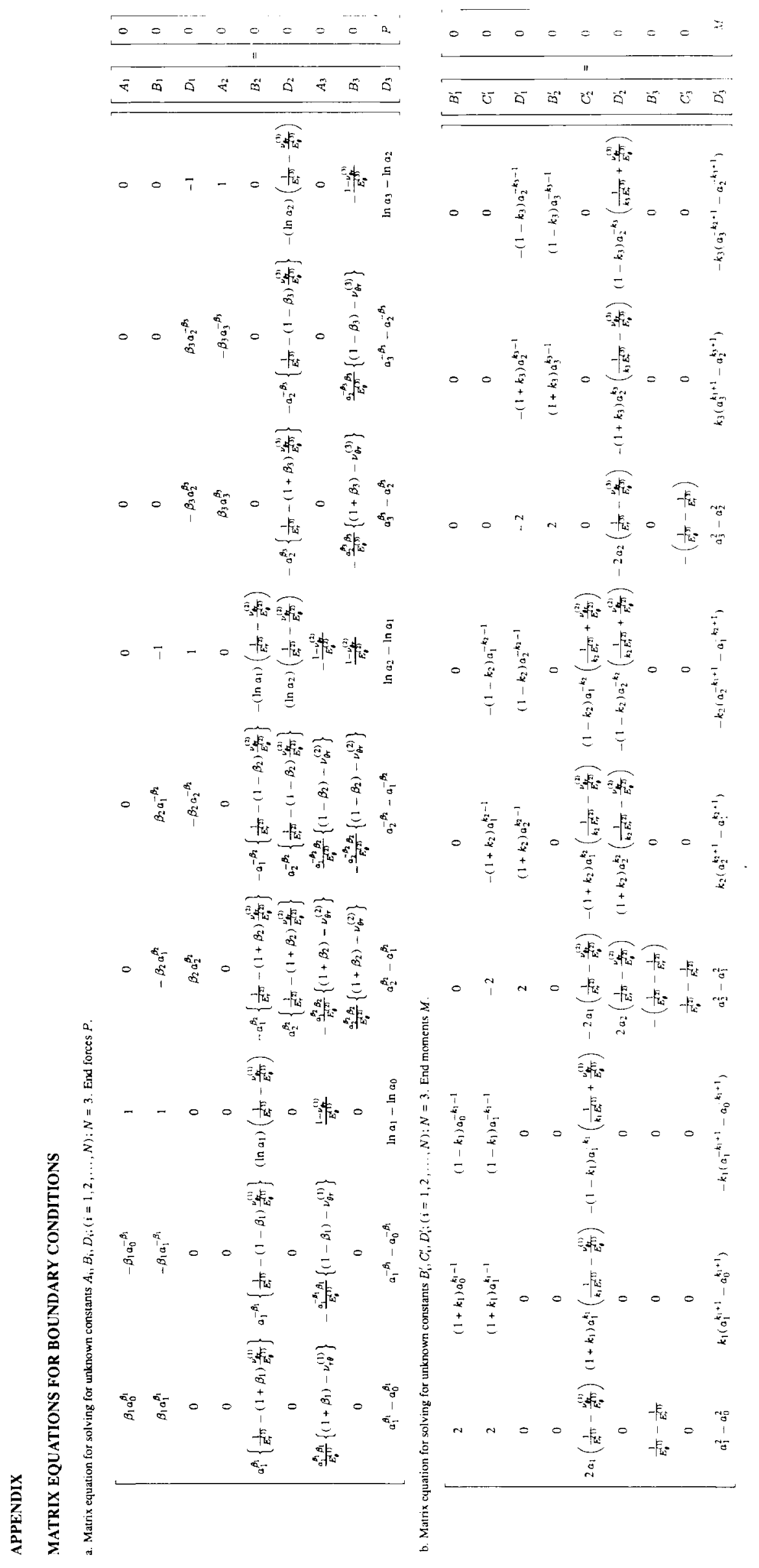





Figure 1. Laminated composite curved-bar test coupon for fatigue delamination study. 




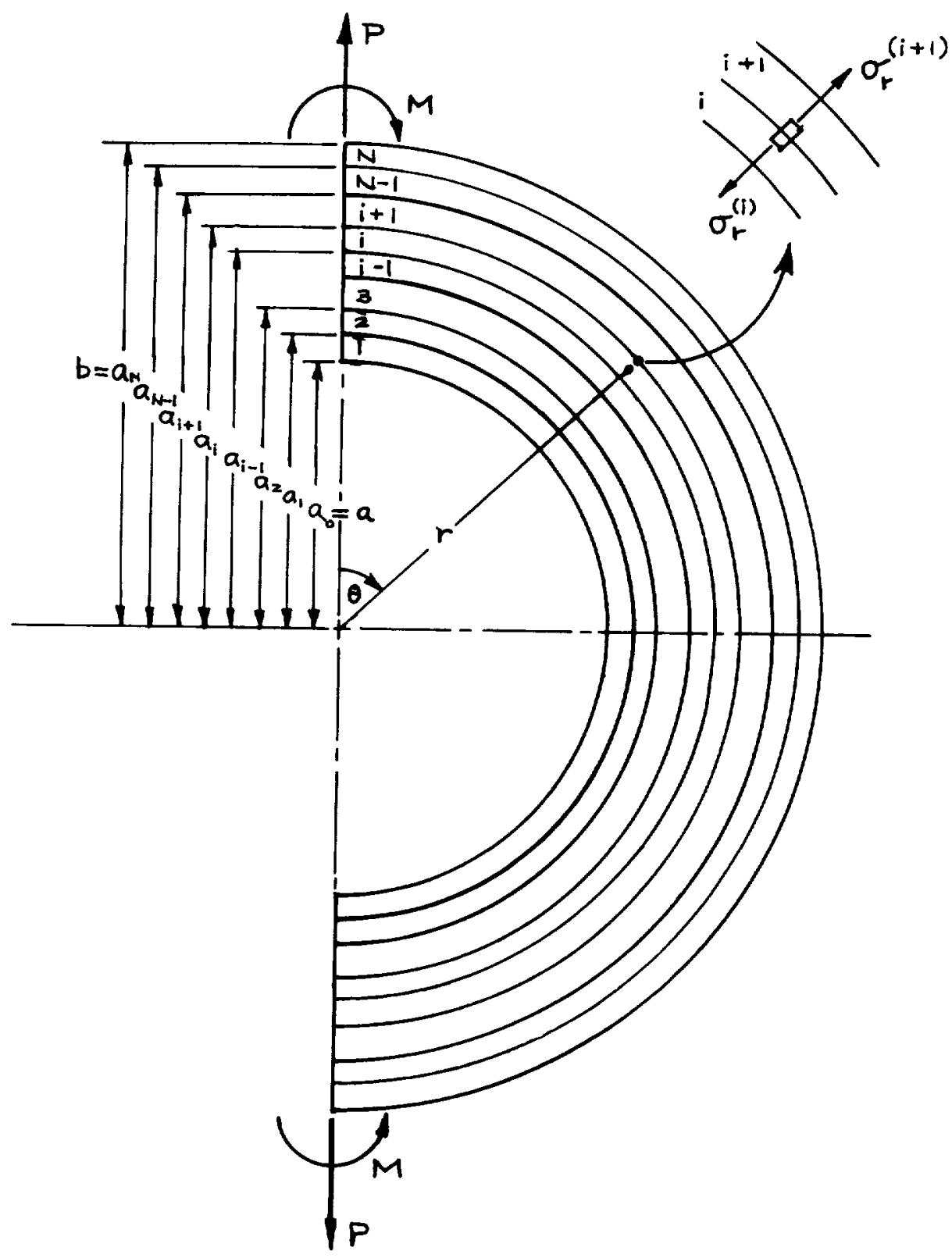

Figure 3. Bending of laminated anisotropic semicircular curved beam by end forces and end moments. 




Figure 4. Finite element model for multilayered semicircular curved bar under bending. 




Figure 5. Finite element model for laminated composite delamination test C-coupon. 


\section{ONENAL PAGE IS \\ OF POOR QUALTY}

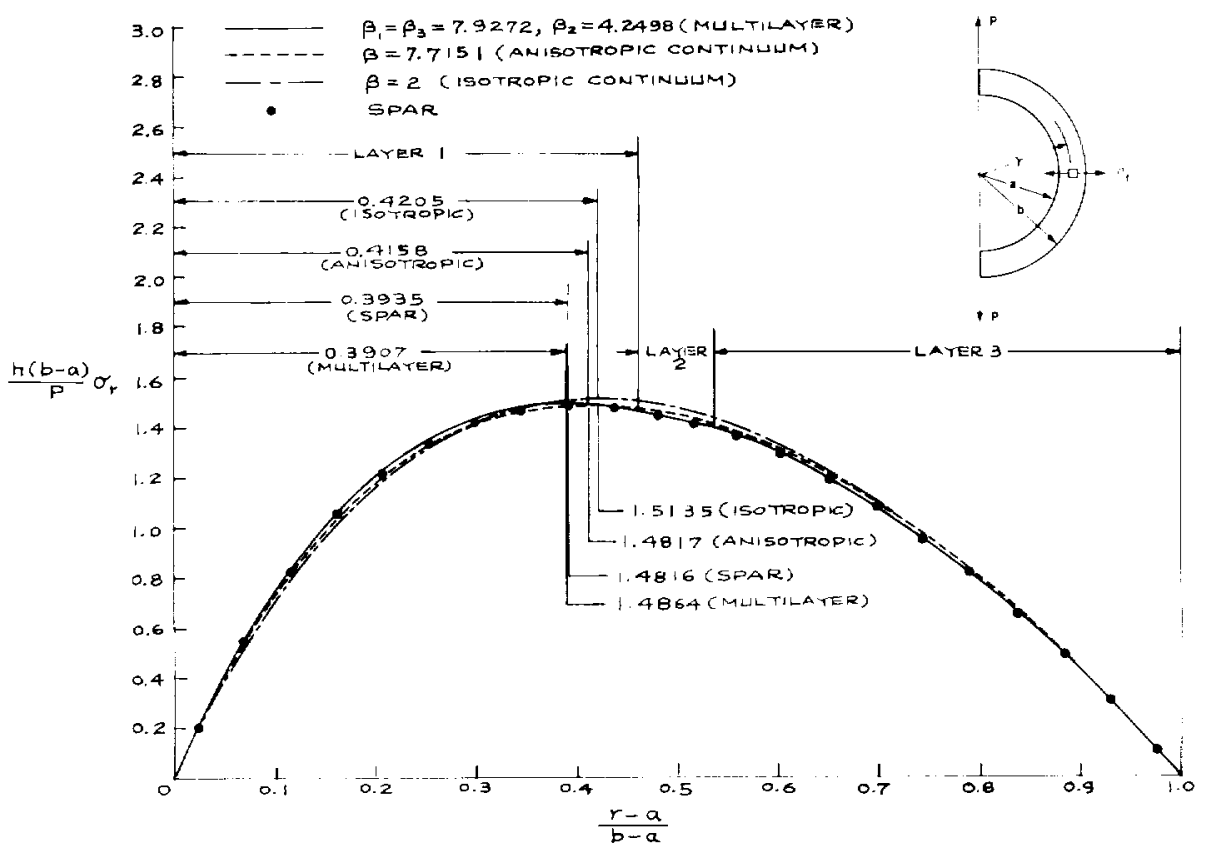

Figure 6. Distribution of radial stress in $\theta=\frac{\pi}{2}$ plane, curved bar under end forces $P ; b / a=1.3767$.

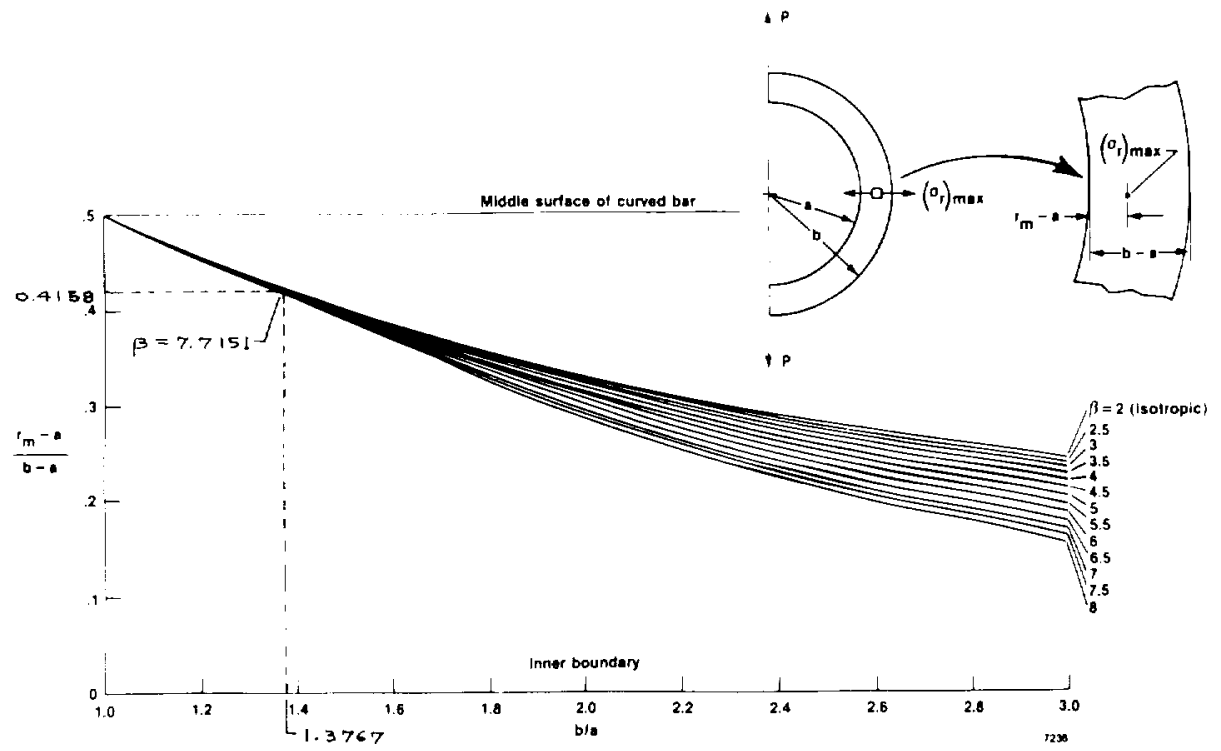

Figure 7. Plots of locations of $\left(\sigma_{r}\right)_{\max }$ as a function of $b / a$ for different values of $\beta$. 


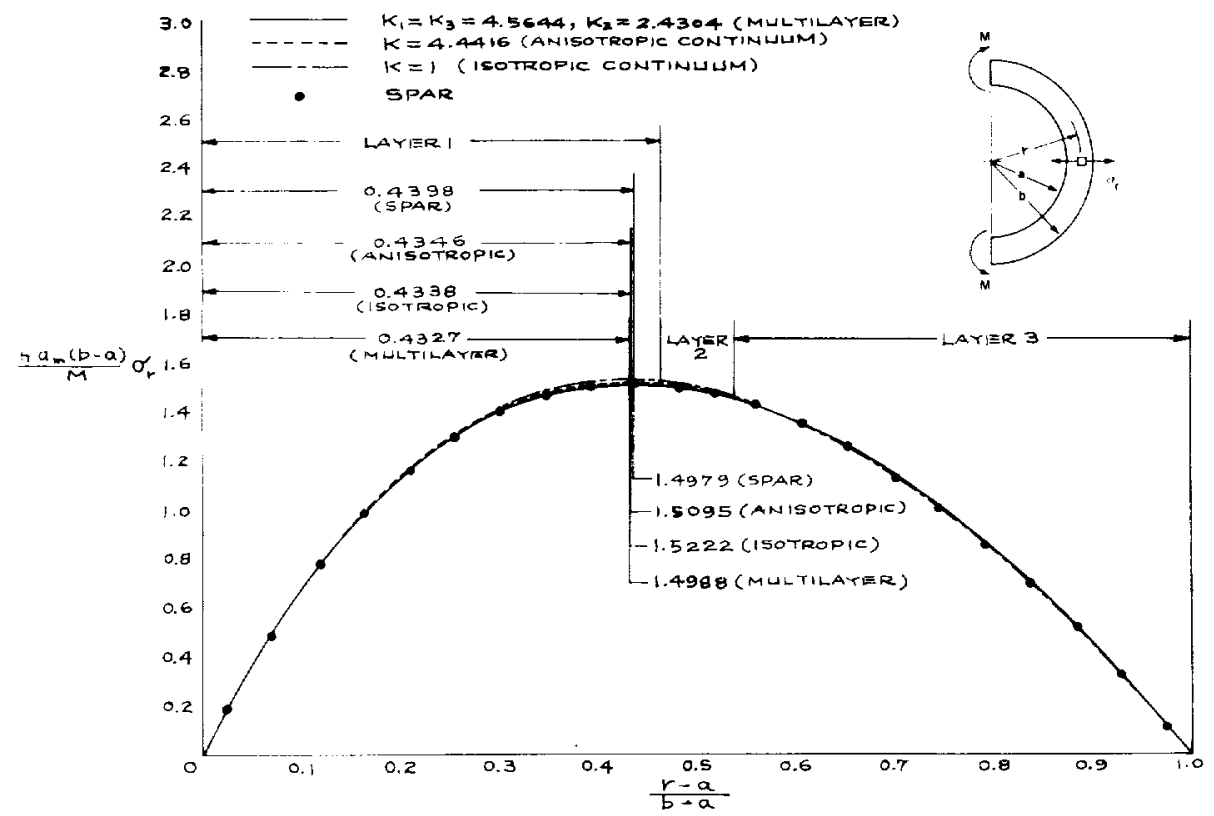

Figure 8. Distribution of radial stress, curved bar under end moments $M ; b / a=1.3767$.



Figure 9. Plots of locations of $\left(\sigma_{\tau}^{\prime}\right)_{\max }$ as a function of $b / a$ for different values of $k$. 


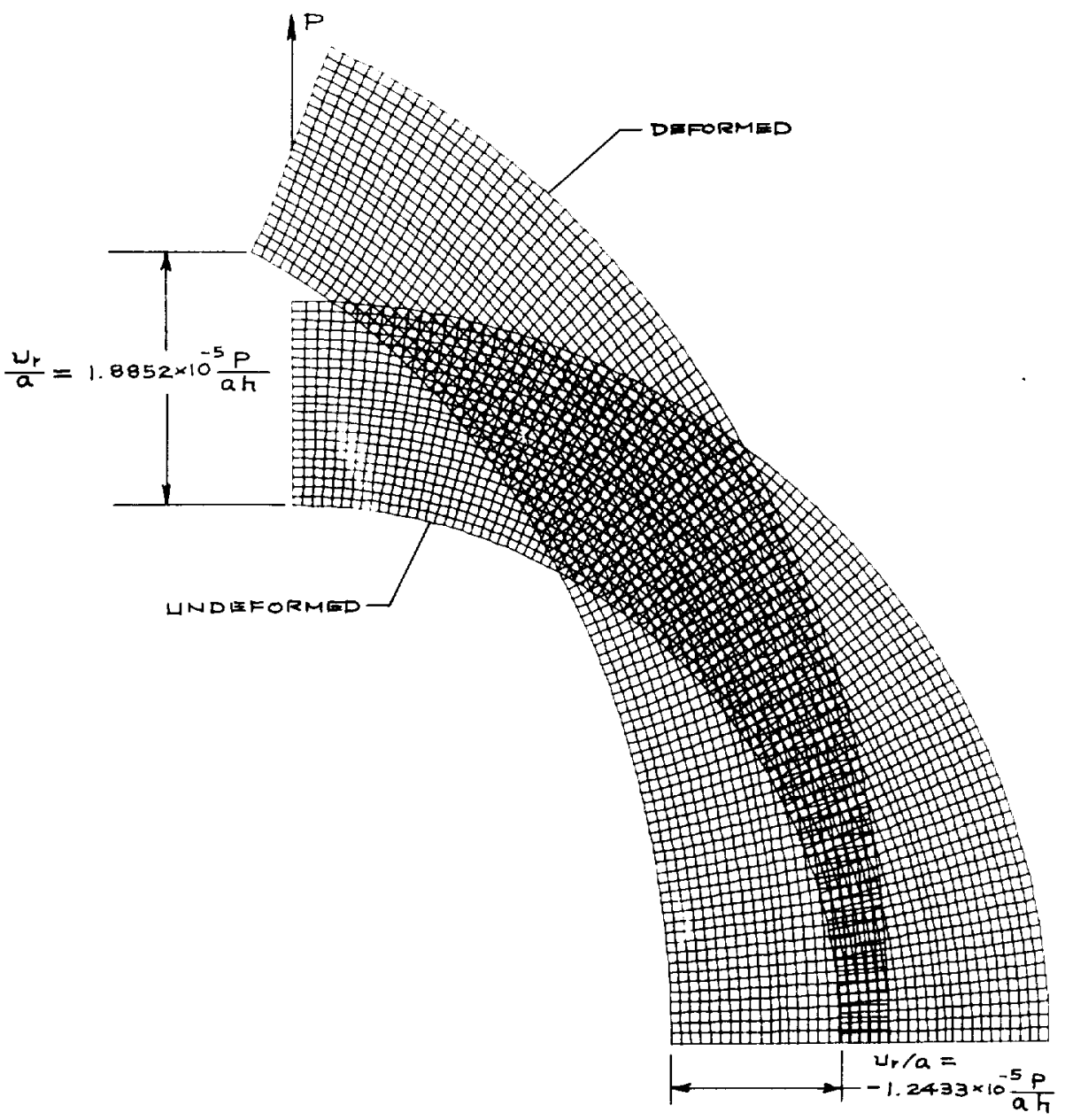

Figure 10. Deformed shape of curved bar under end force $P$. 




Figure 11. Deformed shape of curved bar under end moment $M$. 


\section{ORICNAL PAGE IS \\ OF POOR QUALITY}

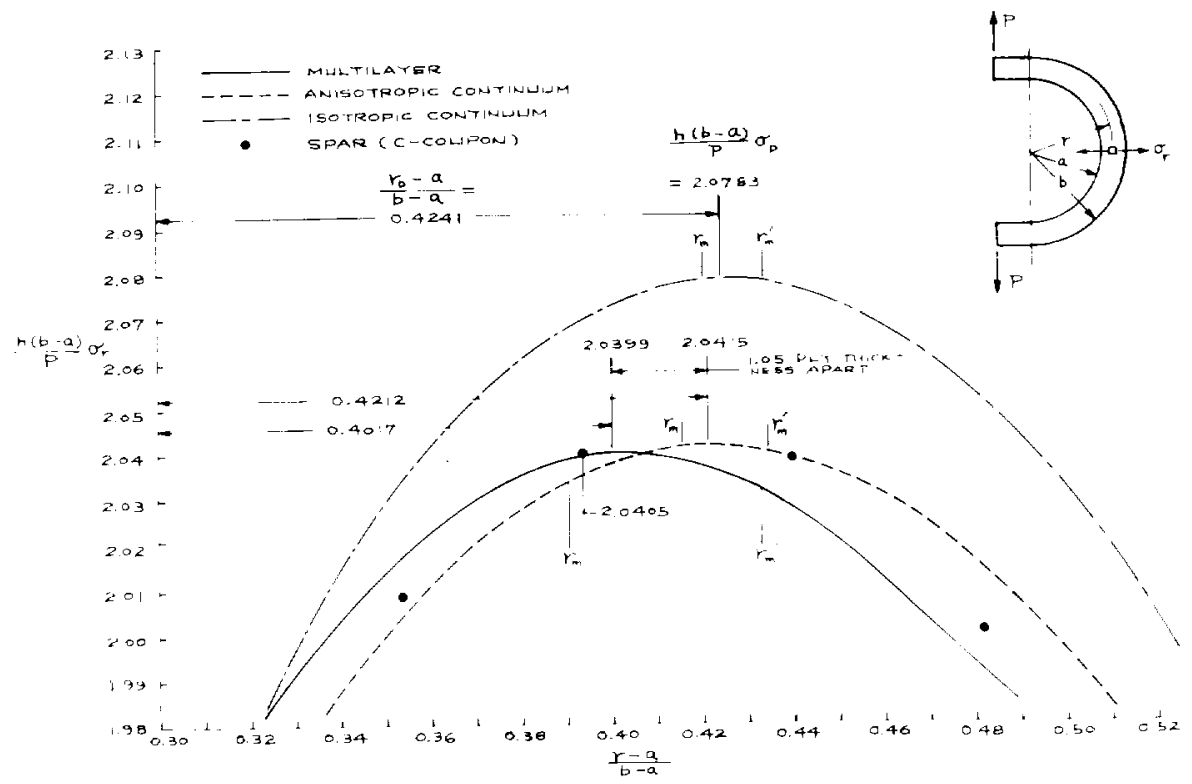

Figure 12. Locations of delamination stress $\sigma_{D}$ in C-coupon predicted from different theories.

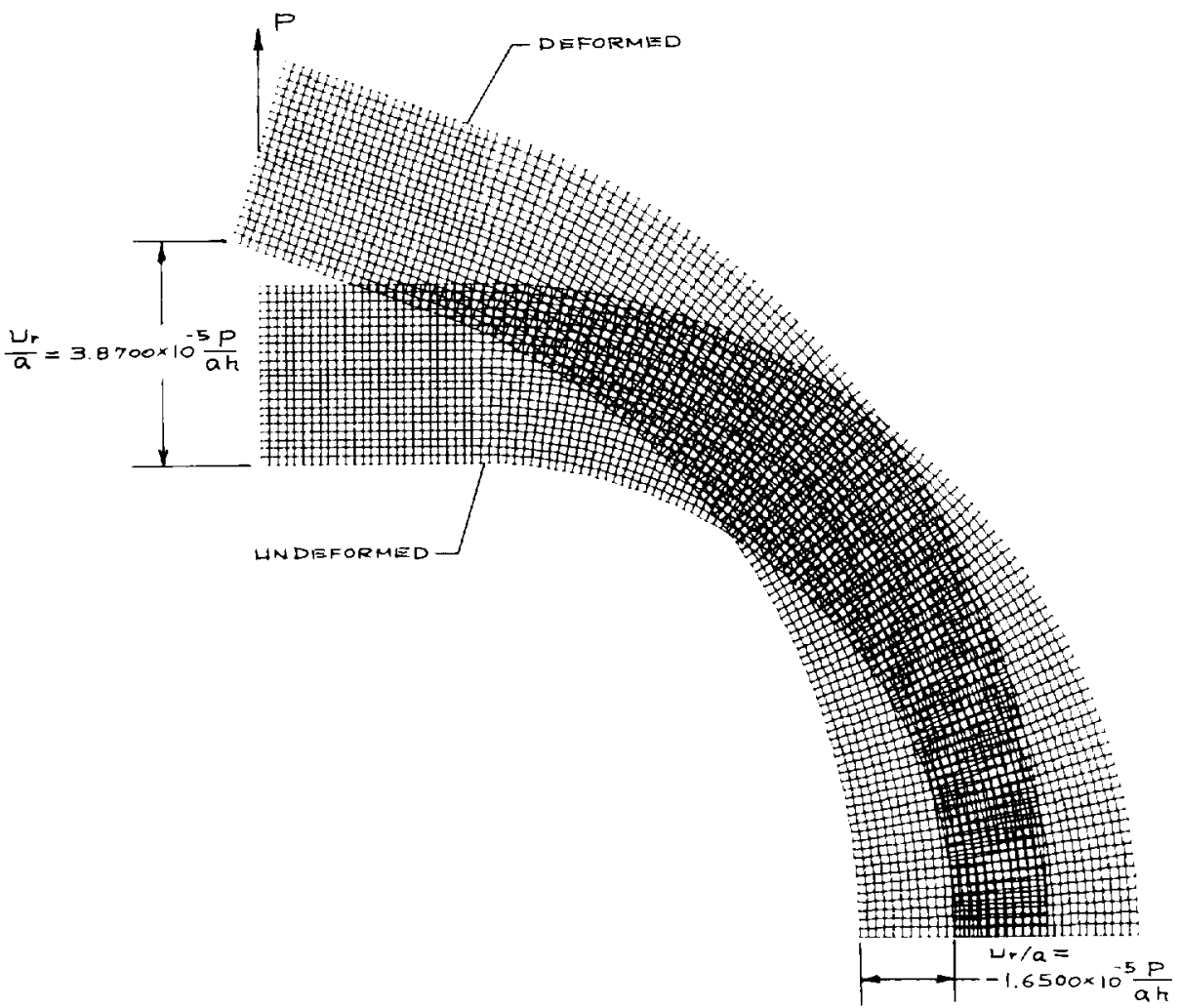

Figure 13. Deformed shape of C-coupon under end forces $P$. 


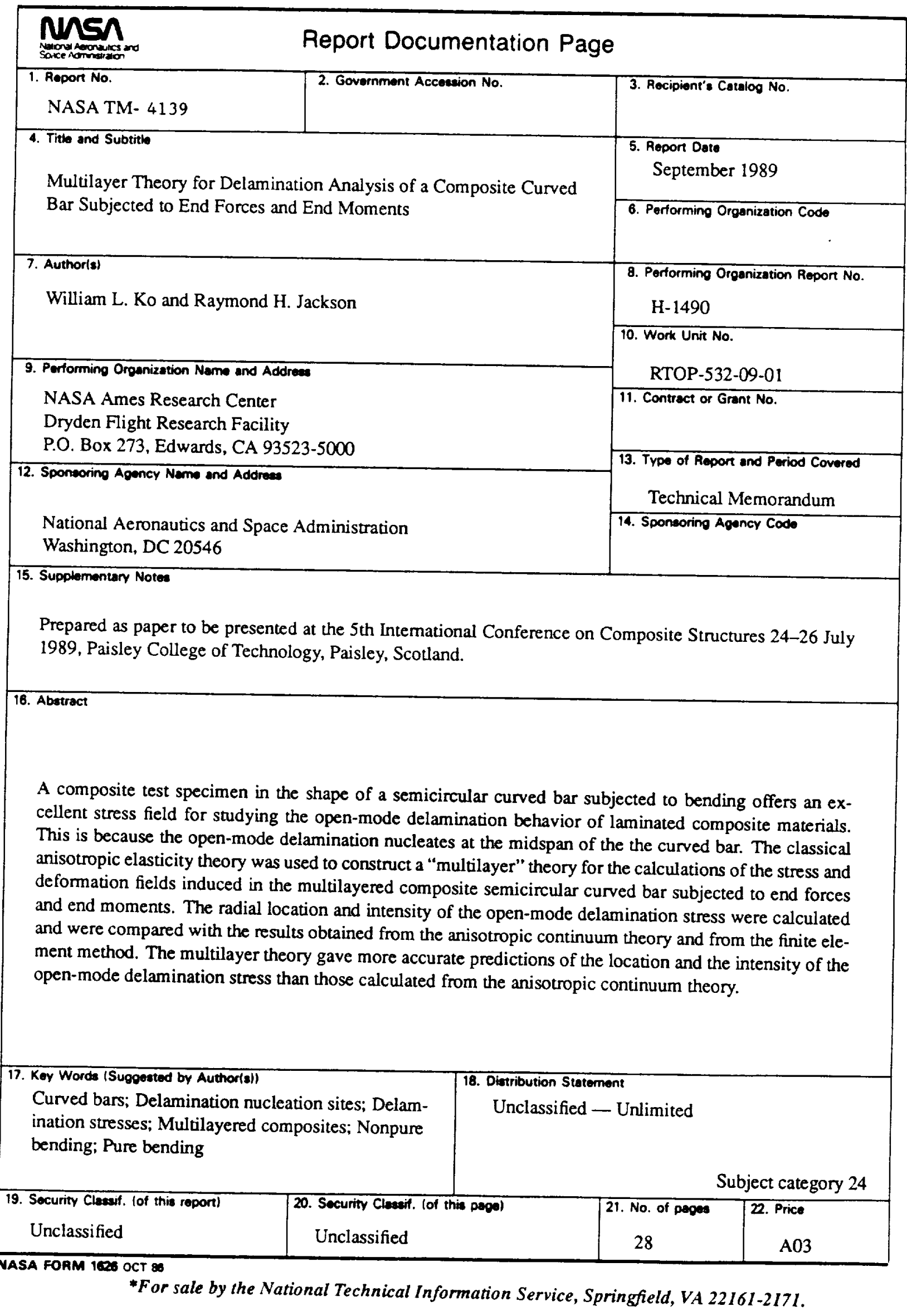

